\title{
Freqüência e intensidade parasitária de helmintos gastrintestinais em bovinos abatidos em frigorífico da região noroeste do Estado de São Paulo, SP, Brasil
}

\author{
Frequency and intensity of infection by helminths in cattle slaughtered at the \\ abattior, of the northwest of region state of São Paulo, Brazil
}

\author{
Katia Denise Saraiva Bresciani ${ }^{1,2}$; Adjair Antônio do Nascimento²; Alvimar José da Costa,2; \\ Alessandro Francisco Talamini Amarante ${ }^{3}$; Silvia Helena Venturolli Perri'; \\ Luis Gustavo Ferraz Lima ${ }^{1}$
}

\begin{abstract}
Resumo: A freqüência e a intensidade parasitária de helmintos gastrintestinais foram estudadas em 48 bovinos, fêmeas, com diferentes graus de cruzamento de raças zebuínas e taurinas, de faixa etária entre 24 a 30 meses, provenientes de oito municípios da região noroeste do Estado de São Paulo e abatidos no Frigorífico Montenegro, Araçatuba, SP, sacrificando-se quatro animais mensalmente, ao longo de um ano. As espécies de helmintos identificadas foram: Cooperia punctata, Cooperia pectinata, Haemonchus similis, Haemonchus placei e Oesophagostomum radiatum. Dos animais examinados, 21 (43,8\%) estavam parasitados por Haemonchus spp. As espécies $H$. similis e $H$. placei foram identificadas isoladamente em igual proporção $(10,4 \%)$ em dez bovinos, sendo que a infecção simultânea foi registrada em outros $11(22,9 \%)$ dos 48 animais. No intestino delgado, 26 bovinos $(54,2 \%)$ apresentaram C. punctata. Dez machos de $C$. pectinata foram identificados em apenas um bovino que albergava também 310 exemplares de $C$. punctata. No intestino grosso, 20 bovinos $(41,7 \%)$ apresentaram O. radiatum. As infecções mistas ocorreram em $52,1 \%$ dos animais, as puras em $12,0 \%$ e $22,9 \%$ dos bovinos não apresentaram helmintos gastrintestinais.

Palavras-chave : helmintos gastrintestinais, bovinos, Haemonchus, Cooperia, Oesophagostomum.
\end{abstract}

Abstract: Parasitary gastrointestinal helminths frequency and intensity were studied in 48 female bovines, with different zebu and taurine crossbreeding degrees, ages ranging between 24 and 30 months, from eight counties in the Northwest of São Paulo State, slaughtered at Frigorífico Montenegro, Araçatuba, São Paulo (Brazil) and four animals were monthly necropsied over one year. The identified gastrointestinal helminth species were: Cooperia punctata, Cooperia pectinata, Haemonchus similis, Haemonchus placei and Oesophagostomum radiatum. Among the 48 animals, 21 (43,8\%) were carrying Haemonchus spp. The Haemonchus similis and the Haemonchus placei species were identified isolated at an equivalent rate $(10,4 \%)$, being simultaneous infection registered in $22,9 \%$ of the cases. In small intestine, 26 bovines $(54,2 \%)$ showed Cooperia punctata. Ten male Cooperia pectinata were identified in only one bovine that also carried 310 Cooperia punctata. In the large intestine, 20 bovines (41,7\%) showed Oesophagostomum radiatum. Mixed infections occurred in $52,1 \%$ of the animals, simple infections in $12 \%$, and $22,9 \%$ of the bovines did not have gastrointestinal helminths.

Key words: gastrointestinal helminths, bovinos, Haemonchus, Cooperia, Oesophagostomum.

\section{Introdução}

Importantes princípios da epidemiologia das helmintoses gastrintestinais de ruminantes foram fundamentados em contribuições de vários autores como Sprent, 1946; Gordon, 1948; Roberts et al., 1952; Lee et al., 1960; Reinecke, 1960; Le Jambre e Royal, 1980; Lichtenfels et al., 1994. Estudos sobre ocorrência de helmintoses gastrintestinais em animais adultos têm sido relatados mundialmente (WARUIRU, et al., 1998; AGNEESSENS et al., 2000; CHOLLET et al., 2000).

No Brasil, inicialmente os autores focalizavam quase que exclusivamente a fauna helmintíca (GRISI e NUERNBERG, 1971). A partir de 1970, com base no conhecimento da epidemiologia dos nematóides gastrin- testinais, foram elaborados consagrados roteiros direcionados ao tratamento estratégico de nematodioses em bovino de corte (PINHEIRO, 1970; BIANCHIN et al., 1996).

Tomando-se a idade como fator determinante na prevalência de helmintos em bovinos, verificou-se que infecções helmintícas eram particularmente incidentes em animais com até 24 meses de idade (ROBERTS et al., 1952; TONGSON e BALEDIATA, 1972). A relação intrínseca entre a intensidade do parasitismo e a faixa etária dos hospedeiros foi citada como um dado epidemiológico essencial em um programa de controle das verminoses gastrintestinais (COSTA et al., 1979).

A maioria das publicações no Brasil disserta sobre o parasitismo em bovinos com idade inferior a um ano

\footnotetext{
1 Departamento de Apoio, Produção e Saúde Animal, FOA/UNESP, Campus de Araçatuba.

${ }^{2}$ CPPAR-Centro de Pesquisas em Sanidade Animal, FCAV/UNESP, Campus de Jaboticabal.

${ }^{3}$ Departamento de Parasitologia do Instituto de Biociências, FMVZ/UNESP, Campus de Botucatu.
} 
(CARNEIRO e FREITAS, 1977; COSTA et al., 1978) entre 3 a 16 meses de idade (COSTA et al., 1971, 1973, 1974; COSTA et al., 1979; OLIVEIRA, 1988; LIMA, 1998; PIMENTEL NETO e FONSECA, 1999).

O desenvolvimento da resposta imune está associado principalmente a uma resposta prévia contra o parasito, que se torna efetiva ao redor de 18 a 24 meses (PADILHA, 1996). Assim, a partir dessa faixa etária, a tendência é de ocorrer redução na carga parasitária, diminuição no número de ovos excretados nas fezes e baixa incidência de casos clínicos de verminose (CHARLES, 1992).

A literatura sobre o parasitismo por nematódeos gastrintestinais em bovinos abatidos em frigoríficos ou estabelecimentos industriais no Brasil é bastante escassa. Machado et al. (1979) avaliaram as infecções por helmintos em 38 bovinos, entre 24-60 meses, procedentes de 19 municípios do Estado de São Paulo, Minas Gerais, Goiás e Mato Grosso, abatidos no Frigorífico Anglo S.A., em Barretos-SP. Foram diagnosticadas nove espécies de nematódeos e uma de cestódeo, perfazendo um total de 4071 helmintos/animal/necropsiado. Em outro estudo, realizado no Matadouro Municipal de Irapuã, Rodrigues et al. (1985) verificaram que 40 bovinos abatidos estavam parasitados por 13 espécies de helmintos, com uma amplitude de infecção média, por animal necropsiado, de 0,25 a 393,75 helmintos

Considerando a importância da região Noroeste do Estado de São Paulo, de grande tradição na pecuária bovina de corte e a escassa informação bibliográfica nacional sobre helmintos de bovinos em idade de abate, realizou-se o presente trabalho com o objetivo de identificar a fauna helmíntica de bovinos abatidos no Frigorífico Montenegro-SP e obter subsídios para que se possa compreender melhor as infecções helmintícas da região.

\section{Material e Métodos}

Após o abate, no Frigorífico Montenegro, Araçatuba, os tratos gastrintestinais de quatro bovinos eram necropsiados mensalmente, durante um ano, no total de 48 animais, pertencentes a diferentes graus de cruzamento, sendo todos fêmeas, com idade variando entre 24 a 30 meses, mantidos em regime extensivo de pastagens e provenientes de propriedades rurais dos municípios de Araçatuba, Birigui, Braúna, Cafelândia, Glicério, Guararapes, Lins e Marília, da Região Noroeste do Estado de São Paulo.

A necropsia parasitológica bem como a colheita e conservação dos helmintos foi realizada de acordo com Costa et al. (1970), sendo levadas ao laboratório de Parasitologia e Doenças Parasitárias do Departamento de Apoio, Produção e Saúde Animal da Faculdade de Odontologia de Araçatuba - UNESP, para posterior contagem e identificação dos helmintos.

O conteúdo de cada frasco foi despejado em uma bandeja de vidro tipo "pirex" e os helmintos aí encontrados foram recolhidos com auxílio de estiletes e colocados em placas de Petri com água. Esta operação foi realizada, sempre que necessária com auxílio de estereomicroscópio. Os parasitos foram separados por gêneros e contados. A identificação específica foi realizada com base nas descrições de Amarante et al. (1997).

A análise estatística dos dados obtidos foi efetuada através do teste qui-quadrado para comparar a proporção de animais infectados ou não por helmintos e do teste qui-quadrado para verificar associação entre ocorrência de parasitos e a estação do ano. As estatísticas foram consideradas significativas quando $p<0,05$. Os dados obtidos foram processados no programa SAS Institute (1988).

\section{Resultados e Discussão}

Os gêneros encontrados nos bovinos com 24 a 30 meses de idade foram Cooperia, Haemonchus e Oesophagostomum. Resultados similares foram observados em Minas Gerais (COSTA et al., 1970; GUIMARÃES, 1971; COSTA et al., 1974), em Goiás (CARNEIRO e FREITAS, 1977), no Mato Grosso (CATTO e UENO, 1981).

As espécies de helmintos gastrintestinais identificadas foram: Cooperia punctata, Cooperia pectinata, Haemonchus similis, Haemonchus placei e Oesophagostomum radiatum.

Nos abomasos dos 48 bovinos examinados, 21 (43,8\%) apresentaram o Gênero Haemonchus e 27 (56,3\%) não possuíam este helminto, com intensidade de 0 a 1660, não sendo considerada significativa essa diferença.

Exemplares de $H$. placei foram detectados em 16 bovinos, em números entre 10 a 420 helmintos, sendo que a grande maioria dos animais (32), correspondentes a $66,68 \%$ do total, não se apresentaram infectados por esta espécie de Haemonchus. Por sua vez, o número mínimo de $H$. similis encontrado foi de 10 atingindo 0 número máximo de 1340 helmintos. Dos 48 bovinos examinados, 32 deles $(66,69 \%)$ não continham $H$. similis no abomaso.

$H$. similis foi tido como a espécie mais freqüente no Mato Grosso (GRISI e NUERNBERG, 1971) e em São Paulo (MACHADO et al., 1979). No Rio de Janeiro, a maior ocorrência foi da espécie $H$. placei (PIMENTEL NETO, 1976). Neste estudo, estas duas espécies foram isoladas individualmente em igual proporção (33,33\%). Em 56,3\% dos animais, não foram encontradas espécies deste parasito no compartimento abomasal (Tabela 1).

Constatou-se maior ocorrência $(20,8 \%)$ de parasitos do gênero Haemonchus no outono e, nos meses de inverno, foram detectadas as menores proporções de parasitos nos bovinos do experimento $(2,1 \%)$; pois este gênero diminuiu em número durante o período seco de acordo com observações de Padilha, 1996. Na primavera, a incidência foi de 8,3\% e no verão 12,5\%. Esses resultados demonstraram que existe associação signifi- 
cativa $(p<0,05)$ entre a presença ou não do parasito e a estação do ano. Já foi estabelecido que as helmintoses ocorriam especialmente nas estações secas (ROBERTS et al., 1952). No entanto, é imprescíndivel mencionar que o índice pluviométrico pode reger o desenvolvimento dos nematódeos nas pastagens e ser fator preponderante na taxa de translação (PADILHA, 1996; AMARANTE et al., 1996).

Nos intestinos delgados, 26 bovinos (54,2\%) apresentaram C. punctata e 22 (45,8\%) não estavam parasitados por nematódeos intestinais. Foram identificados 10 machos de $C$. pectinata em um bovino que albergava simultaneamente 310 exemplares de C. punctata. (Tabela 1). Elevada ocorrência de C. punctata foi descrita por diversos pesquisadores (GRISI e NUEMBERG, 1971; CARNEIRO e FREITAS, 1977; COSTA et al., 1979; MACHADO et al., 1979; CATTO e UENO, 1981; ZOCOLLER et al., 1983; RODRIGUES et al., 1985), assim como é possível que a mesma se adapte melhor a bovinos do que a ovinos (AMARANTE et al., 1997).

Registraram-se de 10 a 2230 parasitos no intestino delgado e notou-se ainda que do total dos 3955 parasitos obtidos, 2530 (64\%) foram isolados no outono e apenas nesta estação do ano os machos foram encontrados em maior quantidade que as fêmeas (34,9\%). Não existiu associação significativa $(p>0,05)$ entre a presença ou não de parasitismo por Cooperia e a estação anual, nesses 48 animais, confirmando estudos que justificam a presença constante deste parasito ao longo do ano devido a sua menor sensibilidade às variações climáticas (REINECKE, 1960; MELO e RIBEIRO, 1977, FURLONG et al., 1985).

Nos intestinos grossos, 20 bovinos (41,7\%) apresentaram O. radiatum com intensidade de 10 e 120 nematóides 28 (58,3\%) não estavam parasitados por formas adultas. Na primavera verificou-se uma proporção de $16,7 \%$ de nematódeos, durante o outono (14,6\%) e no verão uma incidência de $8,3 \%$, indicando correlação positiva entre a existência do parasito e a estação anual. Interessante notar que, no intervalo referente ao inverno, apenas $2,1 \%$ dos bovinos estudados apresentaram positividade para o O. radiatum no intestino grosso.

Infecções mistas ocorreram em $52,1 \%$ dos animais, infecções simples aconteceram em $12 \%$ dos casos e $22,9 \%$ dos bovinos não albergaram parasitas.

\section{Agradecimentos}

Agradecimentos à FUNDUNESP pelo auxílio financeiro para a realização deste trabalho e a Thiago Silva Savietto, Marcelo Eduardo Kondor Nunes e Renato Bazzo Missono pelo apoio recebido.

Tabela 1 - Indicadores de infecções helmintícas em 48 bovinos abatidos em Frigorífico da Região Noroeste do Estado de São Paulo, SP, Brasil.

\begin{tabular}{ccccc}
\hline Espécies & Habitat & $\mathbf{N}^{\mathbf{0}}$ & Intensidade & Freqüência (\%) \\
\hline Haemonchus similis & Abomaso & 16 & $10-1340$ & 33,33 \\
Haemonchus placei & Abomaso & 16 & $10-420$ & 33,33 \\
Cooperia punctata & Intestino delgado & 26 & $10-2230$ & 54,2 \\
Cooperia pectinata & Intestino delgado & 01 & $0-10$ & 2,08 \\
Oesophagostomum & Intestino grosso & 20 & $0-120$ & 41,7 \\
radiatum & & & & \\
\hline
\end{tabular}

\section{Referências Bibliográficas}

AGNEESSENS, J. et al.. Nematode parasitism in adult dairy cows in Belgium. Veterinary Parasitology, Amsterdam, v. 90, n.1-2, p.83-92, 2000.

AMARANTE, A.F.T.; PADOVANI, C.R.; BARBOSA, M. A. Contaminação da pastagem por larvas infectantes de nematódeos gastrintestinais parasitas de bovinos e ovinos em Botucatu - SP. Revista Brasileira de Parasitologia Veterinária, São Paulo, v.5, n.2, p.65-73, 1996.

AMARANTE, A.F.T. et al.. Host specificity of sheep and cattle nematodes in São Paulo state, Brazil. Veterinary Parasitology, Amsterdam, v.73, n.1-2, p.89-104, 1997.

BIANCHIN, I. Epidemiologia e controle de helmintos gastrintestinais em bezerros a partir da desmama, em pasta-

\section{DIMINUIR TABELA}

gem melhorada, em clima tropical do Brasil. Rio de Janeiro - RJ., 1991. 162 f. Tese (Doutorado). Universidade Rural do Rio de Janeiro, Rio de Janeiro, 1991.

BIANCHIN, I. et al.. Epidemiologia dos nematódeos gastrintestinais em bovinos de corte nos cerrados e o controle estratégico no Brasil. Campo Grande: EMBRAPA, 1996, 120p. (Circular Técnica , 24).

CARNEIRO, J.R.; FREITAS, M.G. Curso natural de infecções helmintícas gastrintestinais em bezerros nascidos durante a estação chuvosa em Goiás. Arquivos da Escola de Veterinária, Belo Horizonnte, v.29, n.1, p. 49-62, 1977.

CATTO, J.B., UENO, H. Nematodioses gastrintestinais em bezerros zebus no Pantanal Matogrossense: prevalência, intensidade de infecção e variação estacional. Pesquisa Agropecuária Brasileira,Brasília, v.16, n.1, p. 129-40, 1981. 
CHARLES, T.P. Verminoses dos bovinos de leite. IN: CHARLES, T.P.; FURLONG, J. Doenças parasitárias dos bovinos de leite. Coronel Pacheco: EMBRAPA, CNPGL, 1992. p.55-110.

CHOLLET, J.Y. et al.. Cooperia pectinata and C. punctata, parasites of the abomasum of cattle in northern Cameroon (Central Africa). Veterinary Parasitology, Amsterdam, v.88, n.1-2, p.135-138, 2000.

COSTA, A.J.; NOGUEIRA, C.Z.; COSTA, J.O. Curso natural das helmintoses gastrintestinais em bezerros nascidos durante a estação "seca" em Guaíra, Estado de São Paulo, Brasil. Arquivos do Instituto Biológico, São Paulo, v.45, n.4, p. 291-298, 1978.

COSTA, A.J. et al.. Helmintos parasitos de bezerros do Município de Uruana - Goiás, Brasil. Arquivos da Escola de Veterinária, Belo Horizonte, v..31, n.1, p. 33-36, 1979.

COSTA, H.M.A.; FREITAS, M.G., GUIMARÃES, M.P. Prevalência e intensidade de infecção por helmintos parasitos de bovinos procedentes da área de Três Corações. Arquivos da Escola de Veterinária, Belo Horizonte, v.22, p. 95-101, 1970.

COSTA, H.M.A. et al.. Helmintos parasitos de bezerros procedentes da bacia leiteira de Ibiá, Minas Gerais. Arquivos da Escola de Veterinária, Belo Horizonte, v. 23, n.1, p.221-228, 1971.

COSTA, H.M.A. et al.. Helmintos parasitos de bezerros nabacia leiteira de Calciolândia, Brasil. Arquivos da Escola de Veterinária, Belo Horizonte, v.25, n.2, p. 111-116, 1973.

COSTA, H.M.A. et al.. Variação estacional da intensidade de infecção por helmintos parasitos de bezerros em algumas àreas de produção leiteira em Minas Gerais, Brasil. Arquivos da Escola de Veterinária, Belo Horizonte, v. 26, n. 1, p.143-153, 1974.

FURLONG, J.; ABREU, H.G.L.; VERNEQUE, R.S. Parasitoses dos bovinos na região da Zona da Mata de Minas Gerais 1-Comportamento estacional de nematódeos gastrintestinais. Pesquisa Agropecuária Brasileira, Brasília, v.20, n.1, p.143-153, 1985.

GORDON, H. MacL. The epidemiology of parasitic diseases, with special reference to studies with nematode parasites of sheep. Australian Veterinary Journal, Brunswick, v. 24, p.17-44, 1948.

GRISI, L.; NUERNBERG, S. Incidência de nematóides gastrintestinais de bovinos, no Estado de Mato Grosso. Pesquisa Agropecuária Brasileira: série Veterinária, Brasília, v.6, p.145-149, 1971.

GUIMARÃES, M.P. Variação estacional de larvas infectantes de nematódeos parasitos de bovinos em pastagens de cerrado de Sete Lagoas, Minas Gerais. 1971. 43f. Dissertação (Mestrado) - Instituto de Ciências Biológicas, Universidade Federal de Minas Gerais, Belo Horizonte, 1971.

LEE, R.P.; ARMOUR, J.; ROSS, J.G. The seasonal variations of strongyle infestation in Nigerian Zebu cattle. British Veterinary Journal, London, v.116, n.1, 34-46, 1960.

LE JAMBRE, L..F.; ROYAL, W.M. Meiotic abnormalities in backcross lines of hybrid Haemonchus. Internacional Journal Parasitolology, Elmsford, v.10, n.4, p.281- 286, 1980.

LICHTENFELS, J.R.; PILLIT, P.A.; HOBERG, E.P. New morphological characters of identifyng individual species of Haemonchus spp. (Nematoda - Trichostrongyloidea) and a key to species in ruminants of North America Journal Parasitology, Lancaster, v.80, p.107-109, 1994.

LIMA, W.S. Seasional infection pattern of gastrointestinal nematodes of beef cattle in Minas Gerais State - Brazil. Veterinary Parasitology, Amsterdam, v.74, n.2-4, p.203-214, 1998.

MACHADO, R.Z. et al.. Freqüência e intensidade de infecção por helmintos gastrintestinais em bovinos abatidos no Frigorífico Anglo S.A., Barretos, Estado de São Paulo, Brasil. Científica, Jaboticabal, n. esp., p.103-109, 1979.

MELO, H. J..H.; RIBEIRO, H.S. Helmintos parasitas dos animais domésticos no Estado do Mato Grosso. Arquivos da Escola de Veterinária, Belo Horizonte, v.29, n.2, p.161164, 1977.

OLIVEIRA, G.P. Epidemiologia dos nematoides gastrintestinais em bovinos leiteiros no município de São Carlos, Estado de São Paulo. Pesquisa Agropecuária Brasileira, Brasília, v.23, n.2, p. 1189-1195, 1988.

PADILHA, T. Estratégia para o controle da verminose gastrintestinal de bovinos de leite na região sudeste do Brasil. In: SIMPÓSIO DE CONTROLE DE PARASITOS, 1, 1996. Abstracts... Campinas: [s.n.] 1996. p. 57.

PIMENTEL NETO, M. Epizootiologia da Haemoncose em bezerros de gado de leite no Estado do Rio de Janeiro. Pesquisa Agropecuária Brasileira: série Veterinária, Brasília, v.11, p.101-114, 1976.

PIMENTEL NETO, M.; FONSECA, A.H. Epidemiologia das helmintoses pulmonares e gastrintestinais de bovinos de leite na microrregião homogênea do Vale do Paraíba Fluminenese. A Hora Veterinária, Porto Alegre, v. 19, n.112, p.41- 46, 1999.

PINHEIRO, A. C. Epizootiologia da helmintose de bovinos de Bajé (Rio Grande do Sul) In : CONGRESSO BRASILEIRO DE MEDICINA VETERINÁRIA, 12, 1970, Porto Alegre. Anais... Porto Alegre: [s.n.], 1970. p.247-255.

REINECKE, R.B. A field study on some nematode parasites of bovines in a semi arid area, with special reference to their biology and possible methods of prophylaxis. The Onderstepoort Journal Veterinary Research, Pretoria, v.28, p.365-464, 1960.

ROBERTS, F.H.S.; O' SULLIVAN, P.J. ; RIECK, R.F. The epidemiology of parasitic gastro- enteritis of cattlle. Australian Journal Agricultural Research, Victoria, n.3, v.4, p.1187-1226, 1952.

RODRIGUES, J.; MACHADO, R.Z.; REIS, V.E.A. Freqüência intensidade Parasitária de helmintos gastrintestinais em bovinos abatidos no Matadouro Municipal de Irapuã, SP.,Brasil. Arquivo Brasileiro de Medicina Veterinária e Zootecnia, Belo Horizonte, v. 37, n.3, p.257-263, 1985.

SANTIAGO, M.A.M. Diferença de Haemonchus contortus e Haemonchus placei no Rio Grande do Sul, Brasil. A Hora Veterinária, Porto Alegre, v. 5, n.30, 1986.

SAS INSTITUTE. User's guide : realease 6.03. Cary, 1988. p.1028.

SPRENT, J.F.A. Some observation on the bionomics of Bunostomum phlebotomum (RAILLET, 1900), a hookworm of cattle. Parasitology, London, v.37, n.3-4, p.192-201, 1946.

SVIAVICCO, C.J.S. et al.. Influência das helmintoses 
gastrin-testinais sobre o quadro hematológico de bovinos mestiços holandês $x$ zebu. In: CONGRESSO PANAMERICANO DE CIÊNCIAS VETERINÁRIAS., 15, 1996, Campo Grande. Abstracts... Campo Grande: [s.n.], 1996. p. 324.

TONGSON, M.S., BALEDIATA, E. Epidemiology of bovine parasitic gastroenteritis. Journal of Veterinary Medicine, Berlin, v.11, p.63-72, 1972.

WARUIRU, R.M. et al.. Na abattoir survey of gastrointestinal nematode infections in cattle in the central highlands of Kenya. Veterinary Research Communications, Amsterdam, v.22, n.5, p.325- 334, 1998.

ZOCOLLER, M.C. et al.. Infecção natural por helmintos gastrintestinais em bovinos durante os primeiros dois anos de vida, na região de Ilha Solteira, SP. Arquivo Brasileiro de Medicina Veterinária e Zootecnia, Belo Horizonte, v.35, n.6, p.823-835, 1983. 